Gründen in dieser Frage nur „ein oberflächliches Bild“ (148).

Eine kompakte und gut lesbare Zusammenfassung der Studie findet sich dann als Teil des Resümees am Ende der Arbeit (456-466). Aus der Fülle von Informationen lässt sich z. B. herausheben, dass die befragten Jugendlichen mehrheitlich (68\%) daran glauben, "dass der Konsum von Computerspielen in irgendeiner Weise Auswirkungen auf die Spieler hat" (461). Positive Auswirkungen sind zum Beispiel Denk- und Reaktionsvermögen und Möglichkeiten zum Aggressionsabbau und zur Entspannung. Negative Auswirkungen werden mit den Stichworten „Suchtgefahr, Steigerung des Aggressionspotentials und die Gefahr des Realitätsverlustes" (462) beschrieben. Im Hinblick auf die Werte und den Werttransport im Vergleich zur 15. Shell-Jugendstudie kann die Autorin belegen, dass die Werte "Lebensgenuss“ und "Selbstdurchsetzung " für Jugendliche in Mecklenburg-Vorpommern (MV) wesentlich wichtiger sind als für die Befragten der ShellJugendstudie. Der Wert „Gesetz und Ordnung " ist den Jugendlichen in MV dagegen wesentlich weniger wichtig. Die Ergebnisse Ihrer Studie und die der Shell-Studie seien aber nur sehr bedingt vergleichbar (358).

Der dritte Teil begreift dann die Computerspiele als Herausforderung für den (evangelischen) Religionsunterricht. Nach einer knappen Begründung für die „Öffnung des Religionsunterrichts für das Medium Computerspiel“ (363) präsentiert die Autorin drei Spielanalysen („Black \& White“, „Populous - The Beginnung“, „SIMS“), die parallel aufgebaut sind. Die Spiele werden anhand von drei Kriterien analysiert: 1. religiöse/christliche Elemente im Spiel, 2. „Grundbedürfnisse, Sehnsüchte und Werte Jugendlicher" (363), die im Spiel angesprochen werden, und 3. Themen im Spiel und Anknüpfungspunkte für den Religionsunterricht. Alle drei Spielanalysen enden mit einem Unterrichts- oder Projektvorschlag. Für das Spiel „Black \& White“ wird beispielsweise analysiert, wie dort Werte kommuniziert und Gottesbilder medial geformt werden. Anknüpfungspunkte für den Religionsunterricht bieten sich viele, beispielsweise für die Themen sakrale Räume und Gewissen. Diese Spielanalyse wird abgeschlossen mit einem Unterrichtsvorschlag für die Sekundarstufe I zum Themenkomplex „Gottesbilder, Gottesdarstellungen und -verarbeitungen in den modernen Medien" (Verlaufplan für 2-3 Unterrichtsstunden, Folie, Arbeitsblatt)

Die Arbeit kann belegen, dass Religionsunterricht und moderne, von Jugendlichen selbst favorisierte Computerspiele keinesfalls verschiedenen Welten angehören. Ein sympathisches Gefühl für die Alltagsrelevanz sowohl von Religionsunterricht als auch von Computerspielen trägt sich durch die Arbeit. Dieses Thema umfassend empirisch angegangen und praktisch-theologisch angewendet zu haben, muss der Autorin hoch angerechnet werden. Auch die empirische Studie kann für sich selbst stehen, insoweit hier in Bezug auf eine bestimmte Region Daten zur jugendlichen Nutzung von Computerspielen erhoben wurden. Ein deutlich fokussierterer theoretischer Teil und eine flüssigere Darstellungsform würden die Arbeit aufwerten. Das kompakte und zugängliche Resümee am Ende der Arbeit kann für einen ersten Zugang zum Buch empfohlen werden.

Alexander Filipović

\section{Tilo Hartmann}

\section{Parasoziale Interaktion und Beziehungen}

Baden-Baden: Nomos, 2010. - 131 S.

(Konzepte: Ansätze der Medien- und

Kommunikationswissenschaft; 3 )

ISBN 978-3-8329-4338-7

Die Vorstellung, dass Menschen mit Personen im Fernsehen interagieren und sogar längerfristige Beziehungen zu Medienfiguren aufbauen, scheint für viele auf den ersten Blick etwas gewöhnungsbedürftig. Deshalb macht es Sinn, wenn Tilo Hartmann die beiden Konzepte „Parasoziale Interaktion“ (PSI) und „Parasoziale Beziehung“ (PSB) anhand vieler lebensnaher und gut nachvollziehbarer Beispiele einführt und erklärt. Spätestens wenn er - wie auf Seite 17 - durch die direkte Adressierung des Lesers eine parasoziale Interaktion „provoziert“, sorgt er für einen Aha-Effekt und macht damit die (psychologische) Relevanz dieser beiden Konzepte deutlich. In den 1950er Jahren von Donald Horton, Richard Wohl und Anselm Strauss in zwei häufig zitierten Artikeln theoretisch expliziert, wurden PSI und PSB seit den 1980er Jahren in verschiedenen Disziplinen mit teilweise unterschiedlichem Begriffsverständnis und mit diversen Methoden "beforscht". Hartmann gelingt es, Ordnung in diese Forschungslandschaft $\mathrm{zu}$ bringen. Im umfangreichsten Kapitel 2 nimmt er sich 40 Seiten Zeit, die Entwicklungsgeschichte des Ansatzes nachzuzeichnen. Dabei macht er auf die begrifflichen Unschärfen aufmerksam, die im Zusammenhang mit PSI und PSB immer wieder zu Missverständnissen geführt haben. Ebenso legt 
er für den Leser klar und verständlich die diversen Perspektiven dar, die kulturwissenschaftlich, (medien-)psychologisch und kommunikationswissenschaftlich orientierte Forscher und Forscherinnen auf diese Phänomenen hatten bzw. haben. Das ist gut so, denn es verdeutlicht die Notwendigkeit, parasoziale Interaktionen und parasoziale Beziehungen theoretisch und methodisch von verwandten Konstrukten wie Identifikation oder Involvement abzugrenzen - was in der bisherigen Forschung nicht immer eindeutig passierte. Ebenso notwendig ist es, das Verhältnis von PSI und PSB zu klären und zu einer expliziten Unterscheidung zwischen beiden Konzepten zu gelangen. Beides leistet der Text in aller Klarheit und trägt damit zu einer Präzisierung des theoretischen Verständnisses von PSI und PSB bei.

Immer orientiert an den ursprünglichen Vorstellungen von Horton, Wohl und Strauss werden Gemeinsamkeiten, Abweichungen, Differenzierungen und Weiterentwicklungen der Konzepte dargestellt, erläutert und kommentiert, so dass schließlich ein umfassendes und lebendiges Bild eines Forschungsbereichs entsteht, der sich zentral mit Fragen beschäftigt wie: Was ist das Spezifische an einer medial vermittelten Interaktion im Vergleich zur realen Interaktion mit anderen? Was ist das Besondere an (sozialen) Beziehungen, die daraus entstehen? Welche Faktoren determinieren PSI bzw. PSB? Was sind die Folgen von PSI und PSB? Nach der Lektüre von Kapitel 1 und 2 sollte der Leser erkannt haben, dass diese Fragen keinesfalls trivial sind, sondern eine wissenschaftliche Herausforderung darstellen. Mit Hinweisen auf bislang ungelöste Fragen und Anregungen für weitere wissenschaftliche Untersuchungen in diesem Bereich bietet Hartmann interessante Ansatzpunkte für zukünftige theoretische Diskussion und empirische Forschung. In Kapitel 3 werden die Forschungslogik sowie die verschiedenen Methoden und Instrumente zur Messung von PSI bzw. PSB vorgestellt. Dies führt zu der Erkenntnis, dass die in der bisherigen Forschung verwendeten Messinstrumente nicht frei von Problemen der Inhaltsvalidität waren bzw. sind. Und weil Hartmann es genau nimmt und sich die Mühe gemacht hat, die verschiedenen Verfahren detailliert bis hin zur Formulierung einzelner Items zu beschreiben, kann der Leser jeweils nachvollziehen, ob und wie gut die verwendeten Instrumente mit den jeweiligen theoretischen Voraussetzungen korrespondieren. Die Vielzahl der empirischen Befunde, die in der PSI-/PSB-Forschung bislang erarbeitet wurden, ist in Kapitel 4 gut strukturiert und übersichtlich zusammengefasst.
An dieser Stelle fallen die auch sonst im Buch verwendeten Marginalien als besonders hilfreich auf, denn sie erleichtern die einfache und schnelle inhaltliche Orientierung im Text. Zur guten didaktischen Aufbereitung tragen auch die vielen informativen Kästen zu Kernsätzen, Begriffen, Fallbeispielen, Schlüsselstudien, Akteuren, Anekdoten etc. sowie eine kommentierte Auswahl von wichtigen Studien zu diesem Themenbereich bei. Besonders beeindruckt hat mich die gute Lesbarkeit des Texts mit seinen zahlreichen illustrierenden Beispielen. Gerne hätte ich auch noch meine parasoziale Interaktion mit Kermit getestet, aber dessen Bild ist wohl leider irgendwie verlorengegangen. Die Idee, Lehrbücher auf der Ebene von relevanten „Konzepten“ zu schreiben und damit die Lücke zwischen "großen Überblickswerken" und „Einträgen in Handbüchern und Lexika“ zu füllen, gefällt mir sehr gut. Mit diesem Band zur parasozialen Interaktion und Beziehungen ist sie meines Erachtens hervorragend umgesetzt worden. Wie die Herausgeber der Reihe es sich vorstellen, ist das Buch von Tilo Hartmann für Wissenschaftler und Studierende daher eine empfehlenswerte Bereicherung.

Uli Gleich

\section{Matthias R. Hastall \\ Kommunikation von Gesundheitsrisiken in den Massenmedien}

Der Einfluss von Informations- und

Rezipientenmerkmalen auf die

Botschaftszuwendung und -vermeidung

Baden-Baden: Nomos / Ed. Fischer, 2011.-352

$\mathrm{S}$.

ISBN 978-3-8329-6450-4

(Zugl.: Erfurt, Univ., Diss., 2010)

Die Schweinegrippe, der Dioxin-Skandal oder zuletzt die EHEC-Erkrankungen sind eindrückliche Beispiele, an denen die Relevanz der Frage nach der Kommunikation von Gesundheitsrisiken deutlich wird: Wie können Gesundheitsbotschaften vermittelt werden, um Menschen für Gesundheitsgefahren zu sensibilisieren und ihnen bei informierten Entscheidungen zu helfen, ohne dabei überhöhte Angst und übertriebene Schutzmaßnahmen auszulösen? Matthias R. Hastall widmet sich diesem Thema in der vorliegenden Monographie, die zugleich seine Dissertationsschrift ist, aus medienpsychologischer Perspektive. Ausgehend von der Beobachtung, dass bisherige Studien nur selten (positive) Wirkungen von Gesund- 